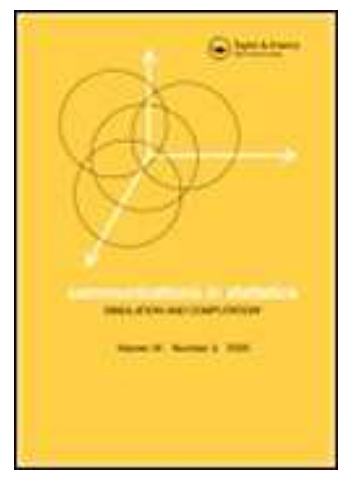

\title{
An improvement of the nonparametric bootstrap test for the comparison of the coefficient of variations
}

\begin{tabular}{|c|c|}
\hline Journal: & Communications in Statistics - Simulation and Computation \\
\hline Manuscript ID: & LSSP-2010-0002.R2 \\
\hline Manuscript Type: & Original Paper \\
\hline $\begin{array}{r}\text { Date Submitted by the } \\
\text { Author: }\end{array}$ & $16-J u l-2010$ \\
\hline Complete List of Authors: & $\begin{array}{l}\text { amiri, saeid; Mathe dept. Uppsala Univ. } \\
\text { Zwanzig, Silvelyn; Mathe dept. Uppsala Univ. }\end{array}$ \\
\hline Keywords: & Bootstrap method, Coefficient of variation, Monte Carlo simulation \\
\hline Abstract: & $\begin{array}{l}\text { In this paper, we propose a new test for examining the equality of } \\
\text { the coefficient of variation between two different populations. The } \\
\text { proposed test is based on the nonparametric bootstrap method. It } \\
\text { appears to yield several appreciable advantages over the current } \\
\text { tests. The quick and easy implementation of the test can be } \\
\text { considered as advantages of the proposed test. The test is } \\
\text { examined by the Monte Carlo simulations, and also evaluated using } \\
\text { various numerical studies. }\end{array}$ \\
\hline \multicolumn{2}{|c|}{$\begin{array}{l}\text { Note: The following files were submitted by the author for peer review, but cannot be converted } \\
\text { to PDF. You must view these files (e.g. movies) online. }\end{array}$} \\
\hline compstat.zip & \\
\hline
\end{tabular}

\section{SCHOLARONE Manuscripts}




\title{
An Improvement of the Nonparametric Bootstrap Test for the Comparison of the Coefficient of Variations
}

\author{
Saeid Amiri* Silvelyn Zwanzig \\ Department of Mathematics, Uppsala University, P.O.Box 480, 75106 Uppsala, Sweden
}

\begin{abstract}
In this paper, we propose a new test for examining the equality of the coefficient of variation between two different populations. The proposed test is based on the nonparametric bootstrap method. It appears to yield several appreciable advantages over the current tests. The quick and easy implementation of the test can be considered as advantages of the proposed test. The test is examined by the Monte Carlo simulations, and also evaluated using various numerical studies.
\end{abstract}

Keywords: Bootstrap method; Coefficient of variation; Monte Carlo simulation.

\section{Introduction}

The coefficient of variation, denoted by $\gamma$ hereafter, is widely calculated and interpreted. It is the ratio of the standard deviation to the mean, a dimensionless measure of the dispersion found to be very useful in many situations. Its application can be found in different disciplines, in the chemical experiments, finance and medical sciences (Nairy and Rao (2003) and Pang et al. (2005)). The sample $\hat{\gamma}$ is the ratio of the sample standard deviation and the sample mean that can be found in most introductory statistics books.

In order to draw inference concerning the population $\gamma$, it is necessary to make assumption on shape of the distribution (Nairy and Rao (2003)). Hence the reported inferences in literature are generally based on parametric models and focus in particular on the $\gamma$ of normal population. It should be noted that the exact distribution of the sample $\hat{\gamma}$ is difficult to obtain even for the normal distribution, even more difficult for skewed distribution (Pang et al. (2005)). An approximation of the distribution of the sample $\hat{\gamma}$ has been considered in Mckay (1932). He showed that $\hat{\gamma}$ has a $\chi^{2}$ distribution if $\gamma<1 / 3$. Mckay's approximation has been considered by many authors (see, for example, Umphery (1983)). Moreover, Forkman and Verill (2008) showed that Mckay's approximation has type II noncentral beta distribution and has asymptotically normal distribution with mean $n-1$ and variance slightly smaller than $2(n-1)$. There are a vast amount of literature on the sample $\hat{\gamma}$ to exploit it in various levels of difficulties (see, for example, Nairy and Rao (2003), Pang et al. (2005), and Mahmoudvand and Hassani (2009) and the references therein).

\footnotetext{
${ }^{*}$ Corresponding author. Tel.: +46(0)184713213; Fax: +46(0)184713201

Email address: saeid@math.uu.se
} 
Nonparametric methods like bootstrap, which free up the analysis from the restrictive assumptions, can be considered as an alternative method. There are few papers that have tried to use bootstrap for the inference of $\hat{\gamma}$; Amiri and Zwanzig (2010) uses the bootstrap exponential tilting to inference one sample $\hat{\gamma}$. In another research, Cabras et al. (2006) proposed a bootstrap test in term of the transformed data to compare the CV of two populations. We consider this idea in the next section. Here we introduce an alternative transformation and consider a different procedure of bootstrap method that leads us to make an improvement of the accuracy of the results from different points of view. As mentioned earlier most of the given tests have aimed $\gamma<1 / 3$ that is necessary condition of Mckay's test, this work will cover the statistical inference of the $\gamma \geq 1 / 3$ as well. This work uses the nonparametric bootstrap approach, since if the chosen distribution is exactly correct then it works well but it suffers when the underlying assumptions are violated, there exists some works that tried to discuss the difference between the parametric and nonparametric bootstrap in other regards (see, for example, Amiri et al. (2010) that consider the comparison of parametric and nonparametric bootstrap of variance).

The paper is organized as follow. The proposed tests based on the bootstrap method are considered in Section 2. Section 3 studies the appropriateness of given methods, and finally the Monte Carlo simulation is used to evaluate the performance of the proposed tests in Section 4 .

\section{The Proposed Tests}

Let $\mathbf{Y}_{\mathbf{i}}=\left(Y_{i 1}, \cdots, Y_{i n_{i}}\right)$ be $i i d$ samples taken from the distribution $F_{i}(i=1,2)$. Consider the following hypothesis;

$$
\begin{aligned}
& H_{0}: \gamma_{1}=\gamma_{2} . \\
& H_{1}: \gamma_{1}>\gamma_{2} .
\end{aligned}
$$

Denote $\gamma=\sigma / \mu$ and $\nu=\mu / \sigma$. The bootstrap methods are the nonparametric resampling that have become a powerful tool for the statistical inference. These methods especially attach a standard error to an estimate and carry out a test of hypothesis. It has been revolutionary for incorporation of computers into statistical practice (see, for example, Efron and Tibshirani (1993), and Davison and Hinkley (1997) and references therein). Here we consider two main approaches; the separate and simultaneous approaches. In the separate approach resampling is done on each sample, whilst in the second method we consider two samples as one sample and then obtain the resamples simultaneously from the new created sample. These approaches are explained in the following.

\subsection{Separate approach}

Consider the following transform to implement a bootstrap test,

$$
\begin{aligned}
& \tilde{Y}_{1 j}=Y_{1 j}^{\prime}-\bar{Y}_{1}^{\prime}+\frac{n_{1} \bar{Y}_{1}^{\prime}+n_{2} \bar{Y}_{2}^{\prime}}{n_{1}+n_{2}}, \quad j=1, \cdots, n_{1}, \\
& \tilde{Y}_{2 j}=Y_{2 j}^{\prime}-\bar{Y}_{2}^{\prime}+\frac{n_{1} \bar{Y}_{1}^{\prime}+n_{2} \bar{Y}_{2}^{\prime}}{n_{1}+n_{2}}, \quad j=1, \cdots, n_{2} .
\end{aligned}
$$


where

$$
Y_{i j}^{\prime}=\frac{Y_{i j}}{S_{i}} S_{p} \quad j=1, \cdots, n_{i}, i=1,2 .
$$

$\bar{Y}_{1}^{\prime}$ and $\bar{Y}_{2}^{\prime}$ are the means of the $\mathbf{Y}_{\mathbf{1}}^{\prime}=\left\{\mathbf{Y}_{\mathbf{1} \mathbf{j}}^{\prime}\right\}$ and $\mathbf{Y}_{\mathbf{2}}^{\prime}=\left\{\mathbf{Y}_{\mathbf{2} \mathbf{j}}^{\prime}\right\}, S_{p}^{2}$ is the pooled sample variance, i.e.,

$$
S_{p}^{2}=\frac{\left(n_{1}-1\right) S_{1}^{2}+\left(n_{2}-1\right) S_{2}^{2}}{n_{1}+n_{2}-2}
$$

where $S_{i}^{2}$ is the sample variance of sample $i$. Indeed, the given transformation allows to resample under $H_{0}$ (the resampling under $H_{0}$ is the essential to the bootstrap test, see Hall and Wilson (1991)), because the sample $\hat{\gamma}$ of $\tilde{Y}_{1 j}$ and $\tilde{Y}_{2 j}$ are equal. The bootstrap steps can be performed as follows;

1. Transform the original data by the transformation $(2)$, and then consider $T_{D}\left(\tilde{\mathbf{Y}}_{\mathbf{1}}^{\prime}, \tilde{\mathbf{Y}}_{\mathbf{2}}^{\prime}\right)=$ $\left|\hat{\gamma}_{1}-\hat{\gamma}_{2}\right| / \sqrt{\frac{\hat{\gamma}_{1}^{2}}{2 n_{1}}+\frac{\hat{\gamma}_{2}^{2}}{2 n_{2}}}$ (the natural approach for the test statistic is the difference of the sample $\hat{\gamma}_{i}$, the proposed statistic is obtained in reformulation in Sachs (1985)), calculate the following statistic

$$
Z_{D}=T_{D} / \sqrt{V_{D}}
$$

where $V_{D}$ is the variance of $T_{D}$, since it is difficult to find, the bootstrap method is used

$$
V_{D}^{*}=\frac{1}{K-1} \sum_{k=1}^{K}\left(T_{D, K}^{*}-\bar{T}_{D}^{*}\right)^{2},
$$

where $K$ is the number of bootstrap replicates and $T_{D, 1}^{*}, \cdots, T_{D, K}^{*}$ are the bootstrap of $T_{D}\left(\tilde{\mathbf{Y}}_{\mathbf{1}}^{\prime}, \tilde{\mathbf{Y}}_{\mathbf{2}}^{\prime}\right)$. 2. Generate samples with replacement of $\tilde{\mathbf{Y}}_{1}^{\prime}$ and $\tilde{\mathbf{Y}}_{2}^{\prime}$, separately. Denote the resamples by $\left(\tilde{\mathbf{Y}}_{1 b}^{*}, \tilde{\mathbf{Y}}_{2 b}^{*}\right), b=1, \cdots, B$, and calculate

$$
Z_{D}\left(\tilde{\mathbf{Y}}_{1 b}^{*}, \tilde{\mathbf{Y}}_{2 b}^{*}\right)
$$

3. Handle the replications as iid random samples and calculate the $p$-value of $H_{0}: \gamma_{1}=\gamma_{2}$ vs $H_{1}: \gamma_{1}>\gamma_{2}$ by

$$
p-\text { value }=\frac{\#\left\{Z_{D}\left(\tilde{\mathbf{Y}}_{1 b}^{*}, \tilde{\mathbf{Y}}_{2 b}^{*}\right)>Z_{D}\left(\tilde{\mathbf{Y}}_{1}, \tilde{\mathbf{Y}}_{2}\right)\right\}+1}{B+1}
$$

The complete discussion can be found in Cabras et al. (2006). In the rest of the paper, we consider the abbreviation $B I$ for this test. 


\subsection{Simultaneous approach}

Now, we consider the two samples simultaneously. Consider two transformations $\mathcal{Y}_{i j}=Y_{i j} / \bar{Y}_{i}$ and $\mathcal{X}_{i j}=Y_{i j} / S_{i}$, the division of observations by the mean and standard deviation, they are achieved to get the appropriate tests for $\gamma$. The steps of bootstrap test can be as follow. 1. Transform original data using $\mathcal{Y}_{i j}=Y_{i j} / \bar{Y}_{i}$, and then calculate the following statistic

$$
T\left(\mathcal{Y}_{1}, \mathcal{Y}_{2}\right)=S\left(\mathcal{Y}_{1}\right)-S\left(\mathcal{Y}_{2}\right)=\frac{S_{1}}{\bar{Y}_{1}}-\frac{S_{2}}{\bar{Y}_{2}} .
$$

2. Generate samples with replacement of $F_{n}(y)=\frac{1}{n} \sum_{i=1}^{2} \sum_{j=1}^{n_{i}}\left(\mathcal{Y}_{i j} \leq y\right)$, where $n=n_{1}+n_{2}$, actually $F_{n}(y)$ is the empirical distribution of the pooled observations into one sample. Let denotes the resampling by $\left(Y_{1 b}^{*}, Y_{2 b}^{*}\right), \quad b=1, \cdots, B$, and calculate

$$
T_{b}\left(\mathcal{Y}_{1 b}^{*}, \mathcal{Y}_{2 b}^{*}\right)=S\left(\mathcal{Y}_{1 b}^{*}\right)-S\left(\mathcal{Y}_{2 b}^{*}\right) .
$$

3. Handle the replications as iid random samples and calculate the p-value of $H_{0}: \gamma_{1}=\gamma_{2}$ vs $H_{1}: \gamma_{1}>\gamma_{2}$ by

$$
p-\text { value }=\frac{\#\left\{T_{b}\left(\mathcal{Y}_{1 b}^{*}, \mathcal{Y}_{2 b}^{*}\right)>T\left(\mathcal{Y}_{1}, \mathcal{Y}_{2}\right)\right\}}{B+1}
$$

This procedure should be used for $\mathcal{X}_{i j}$ and $\tau\left(\mathcal{X}_{1 j}, \mathcal{X}_{2 j}\right)=\overline{\mathcal{X}}_{1}-\overline{\mathcal{X}}_{2}=\bar{Y}_{1} / S_{1}-\bar{Y}_{2} / S_{2}$. The proposed tests based on the $T($.$) and \tau($.$) are referred to as the B I I$ and $B I I I$, respectively.

\section{Appropriateness of tests}

Often the statistical test is expressed by calculating a p-value that is the probability, under holding $H_{0}$, that a given test statistic $T$ exceeds its observed value. If the statistical test and its distribution are correct then the p-value is readily obtained and it has a uniform distribution under holding $H_{0}$. Robins et al. (2000) discuss the effect of the unknown parameter on the p-value using theorems and examples, they show when the mean of test statistic $T$ depends on $\theta$, the plug-in p-value is conservative, since the bootstrap method is based on plug-in principle, this condition is necessary to study. Since the parameter of interest is $\gamma$, the nuisance parameters are $\mu$ and $\sigma$. This is considered by Cabras et al. (2006) to study the appropriateness of $B I$, they consider the mean (and variance) under null hypothesis, $H_{0}$, that should not depend on population parameters, i.e.,

$$
E_{H_{0}}\left(T \mid \mu_{1}, \mu_{2}, \sigma_{1}, \sigma_{2}\right)=E_{H_{0}}(T) .
$$

They study this condition using simulations. Here aim is to examine the appropriateness of the new proposed tests, $B I I$ and $B I I I$. In order to study the uniformity of p-value under the null hypothesis, Monte Carlo experiments implemented with 1000 simulations were used. The number of bootstrap replications was $B=500$. The Q-Q plot is given in Figure 1 for different 

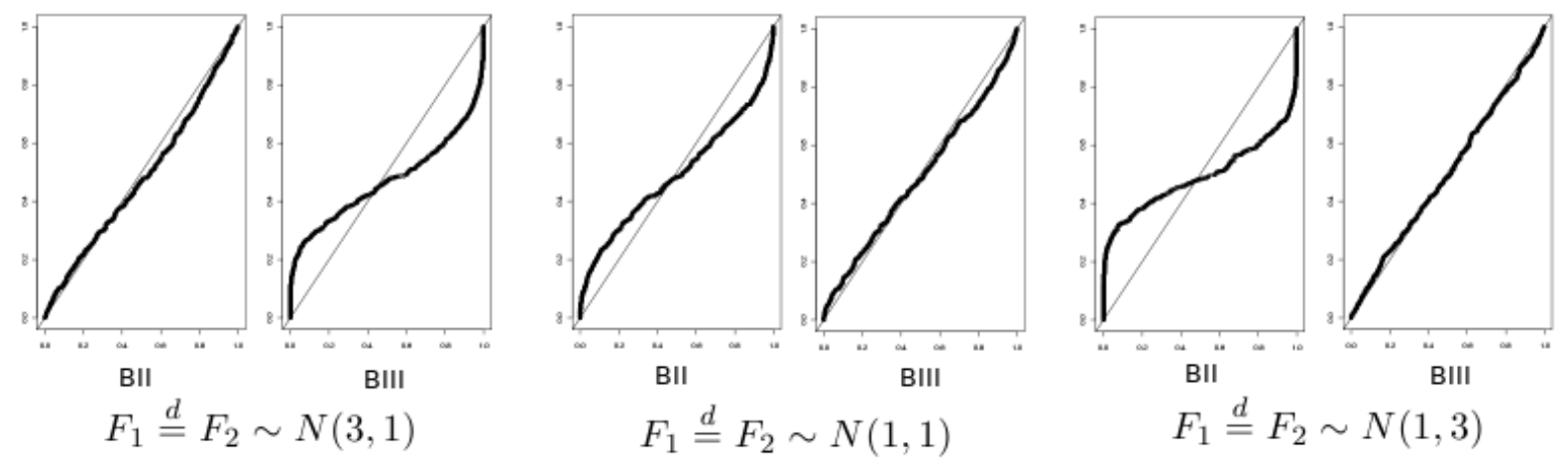

Figure 1: Q-Q plot of the simulated p-value for the method BII and BIII.

values of $\gamma(\gamma=1 / 3,1,2)$. Here only the result of $n=30$ is given. However, the results are examined for $n \geqslant 5$. The results clearly indicate that for $\gamma=1 / 3$, the p-value of $B I I$ has the uniform distribution whilst for $\gamma=1,2$, the p-value of $B I I I$ is uniformly distributed. Therefore, a composite statistics, denotes as $B C$, can be nominated i.e.,

$$
B C=\left\{\begin{array}{cc}
B I I & \gamma<0.6 \\
B I I I & \gamma \geq 0.6
\end{array}\right.
$$

for the test $H_{0}: \gamma_{1}=\gamma_{2}$ vs $H_{1}: \gamma_{1}>\gamma_{2}$. The value of 0.6 is approximated by simulating a ranges of normal distributions with different $\gamma$, around the value of 0.6 the $B I I$ test and $B I I I$ test have the closed p-values. Since the $\gamma$ is unknown the $\hat{\gamma}$ can be used, the $\left(\hat{\gamma}_{1}+\hat{\gamma}_{2}\right) / 2$ can be a suitable criterion to decide which test should be used.

In order to study the second property, consider the following statements that are discussed by Nairy and Rao (2003),

$$
\begin{aligned}
& E(\hat{\gamma})=\gamma+\frac{1}{n}\left(\gamma^{2}-\frac{1}{4}\right)+O\left(n^{-2}\right) . \\
& E(\hat{\nu})=\nu+\frac{3 \nu}{4 n}+O\left(n^{-2}\right) .
\end{aligned}
$$

where $\hat{\gamma}=s / \bar{x}$ and $\hat{\nu}=1 / \hat{\gamma}$. In virtue of the above statements, can show the expected value of the proposed statistics are not in term of the nuisance parameters,

$$
\begin{aligned}
E_{H_{0}}(\hat{T}) & \approx 0 . \\
E_{H_{0}}(\hat{\tau}) & \approx 0 .
\end{aligned}
$$

\section{Comparison with the existing method}

Let us now evaluate the performance of the proposed tests using Type I error and the statistical power. We used the Monte Carlo experiments in order to study finite sample properties of the tests, implemented by 1000 simulations. The proposed tests are based on the same simulated data that increase the accuracy of the comparison results. The bootstrap resampling was performed by $\mathrm{B}=500$. In order to making a comparative evaluation of testing procedures, we seek 
Table 1: The significance level of the simulated p-values at $\alpha=0.10$

\begin{tabular}{lcccccc}
\hline Underlying & & \multicolumn{5}{c}{$n$} \\
\cline { 3 - 7 } distribution & $\gamma$ & test & 10 & 20 & 30 & 50 \\
\hline$F_{1} \stackrel{d}{=} F_{2} \sim N(3,1)$ & $\frac{1}{3}$ & $B I$ & $\mathbf{0 . 0 8 3}$ & $\mathbf{0 . 1 0 1}$ & $\mathbf{0 . 0 9 5}$ & $\mathbf{0 . 1 0 2}$ \\
& & $B I I$ & $\mathbf{0 . 1 1 5}$ & $\mathbf{0 . 1 3 0}$ & $\mathbf{0 . 1 1 9}$ & $\mathbf{0 . 1 3 1}$ \\
& & $B I I I$ & 0.250 & 0.302 & 0.305 & 0.299 \\
$F_{1} \stackrel{d}{=} F_{2} \sim N(1,1)$ & 1 & $B I$ & 0.005 & 0.013 & 0.051 & 0.080 \\
& & $B I I$ & 0.224 & 0.245 & 0.225 & 0.244 \\
& & $B I I I$ & $\mathbf{0 . 1 3 5}$ & $\mathbf{0 . 1 4 3}$ & $\mathbf{0 . 1 5 1}$ & $\mathbf{0 . 1 5 2}$ \\
$F_{1} \stackrel{d}{=} F_{2} \sim N(1,3)$ & 3 & $B I$ & 0.041 & 0.031 & 0.015 & 0.014 \\
& & $B I I$ & 0.335 & 0.339 & 0.329 & 0.346 \\
& & $B I I I$ & $\mathbf{0 . 1 0 2}$ & $\mathbf{0 . 1 0 8}$ & $\mathbf{0 . 1 2 6}$ & $\mathbf{0 . 1 2 1}$ \\
\hline
\end{tabular}

Since the ratio of the mean and standard variation is of interest not their values separately, the distribution of p-value of the test of $H_{0}: \gamma_{1}=\gamma_{2}$ vs $H_{1}: \gamma_{1}>\gamma_{2}$ where $\left(F_{1} \sim N(3,1), F_{2} \sim\right.$ $N(4.5,1.5)),\left(F_{1} \sim N(1,1), F_{2} \sim N(1.5,1.5)\right)$ and $\left(F_{1} \sim N(1.5,4.5), F_{2} \sim N(1,3)\right)$ for the sample size of 30 are given in Figure 3, they admit that the tests consider the ratio of $\mu$ and $\sigma$. Table 2 represents the statistical power, it includes the simulated p-value where the underlying distributions are $\left(F_{1} \sim N(3,1), F_{2} \sim N(3,2)\right),\left(F_{1} \sim N(1,1), F_{2} \sim N(1,2)\right)$ and $\left(F_{1} \sim N(1,2), F_{2} \sim N(1,3)\right)$, respectively. The results indicate that the proposed tests have sufficient power to diagnosis the incorrect null hypothesis.

\section{Conclusion}

We proposed new test for assessing the equality of two coefficient of variations. The proposed test is based on alternative transformation of the original data and different bootstrap procedures. The performance of the existing bootstrap test was also compared with the proposed bootstrap tests. The appropriateness of the new test was discussed. It is shown that the composite test $(B C)$ can be considered as a suitable test for the comparison of the $\gamma$. The results with strong evidence indicate that the $B C$ test has a good performance according to the values of Type I 

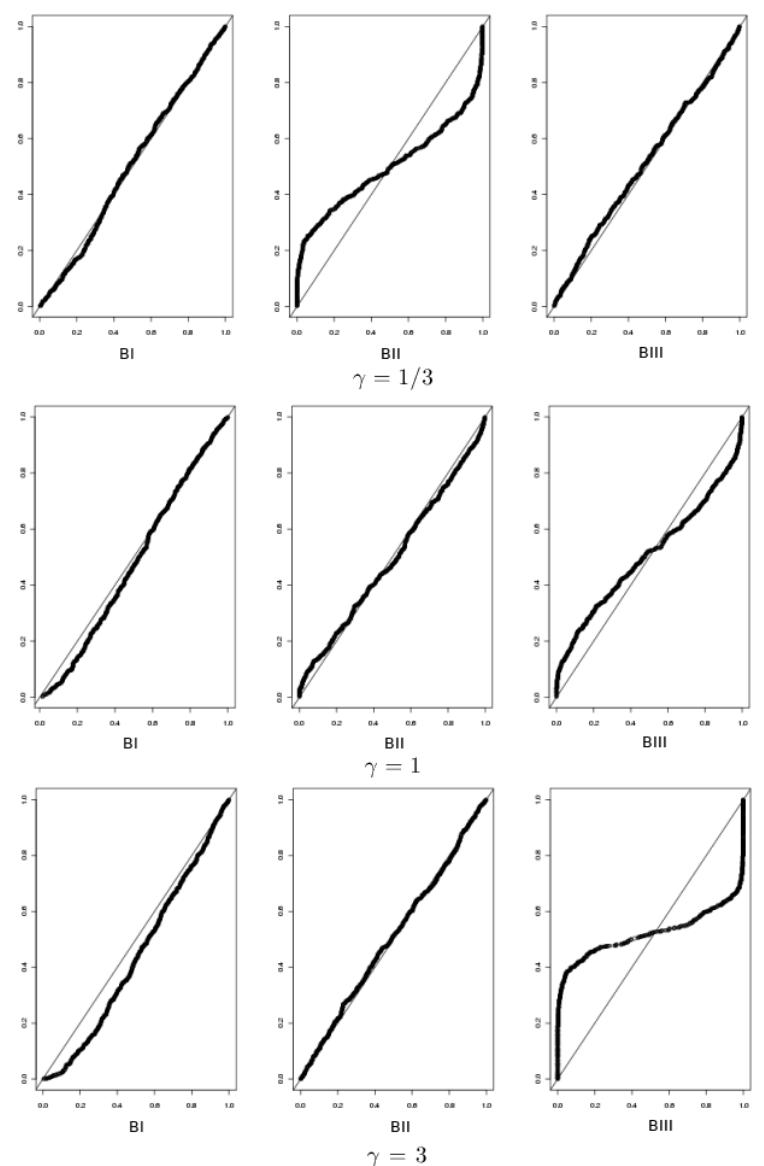

Figure 2: Q-Q plot of the simulated p-value for the method BII and BIII.

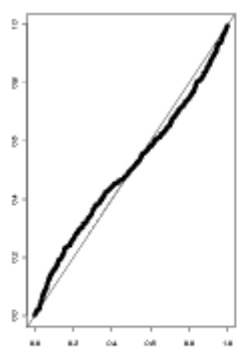

BII

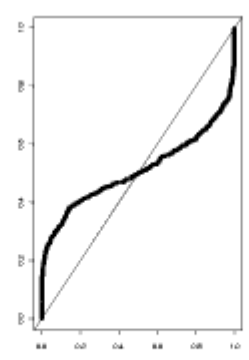

BIII

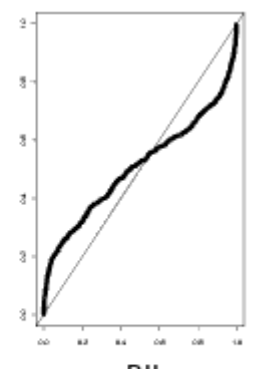

BII

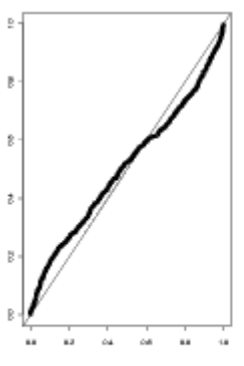

BIII

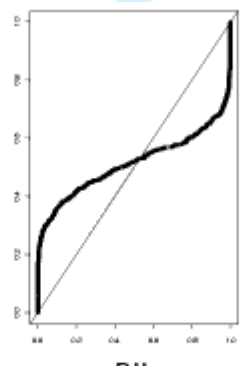

BII

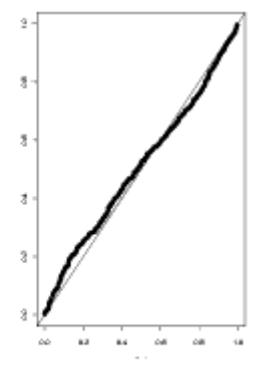

BIII

$\gamma=1 / 3$ $\gamma=3$

Figure 3: Q-Q plot of the simulated p-value with different $\mu$ and $\sigma$ but the same $\gamma$. 
Table 2: Power comparison of various tests at the level $\alpha=0.10$

\begin{tabular}{lccccc}
\hline Underlying & & \multicolumn{4}{c}{$n$} \\
\cline { 3 - 6 } distribution & test & 10 & 20 & 30 & 50 \\
\hline$\left(F_{1} \sim N(3,1), F_{2} \sim N(3,2)\right)$ & $B I I$ & 0.631 & 0.863 & 0.959 & 0.994 \\
& $B I I I$ & 0.606 & 0.874 & 0.962 & 0.994 \\
$\left(F_{1} \sim N(1,1), F_{2} \sim N(1,2)\right)$ & $B I I$ & 0.124 & 0.249 & 0.472 & 0.743 \\
& $B I I I$ & 0.338 & 0.535 & 0.682 & 0.827 \\
$\left(F_{1} \sim N(1,2), F_{2} \sim N(1,3)\right)$ & $B I I$ & 0.155 & 0.286 & 0.390 & 0.595 \\
& $B I I I$ & 0.266 & 0.392 & 0.484 & 0.616 \\
\hline
\end{tabular}

error and statistical power. An advantage of the suggested test is that it is much faster than the existing bootstrap test.

It is noteworthy that the results hold greatly for the normal and mixture normal distribution and other distribution tending to the normal distribution, but for distribution rather than normal distribution with $\gamma>1 / 3$ (the violation of Mckay's condition), the accuracy of the proposed test maybe suffered.

Moreover, regardless of the advantages and disadvantages of the mentioned tests, this work can be considered as a practice in the bootstrap method to inference of the $\gamma$ : An appropriate transformation of the observations used by Cabras et al. (2006) to allow the separate resampling, inversely this paper uses another simple transformation with the simultaneous resampling.

Because of the applicability of inference $\hat{\gamma}$, a library in $\mathbf{R}$ language, $\mathbf{B C V}$, including the proposed tests and other methos is under construction by authors and available upon request.

\section{Acknowledgements}

The authors would like to thank the editor and the referees for their constructive comments. The authors would like to express their sincere appreciation to S. Cabras (Cagliari Univ.) for correcting the commands of $B I$ test. The authors are grateful to H. Hassani (Cardiff Univ.) for useful suggestions.

\section{References}

[1] Amiri, S., Zwanzig, S. (2010). Statistical Inference of the Coefficient of Variation using Exponential Tilting. Mathematics Dept. Uppsala University.

[2] Amiri, S., von Rosen, D., Zwanzig, S. (2010). A Comparison of Bootstrap Methods for Variance Estimation. To appear in Journal of statistical theory and applications.

[3] Cabras, S., Mostallino, G., Racugno, W. (2006). A Nonparametric Bootstrap Test for the Equality of Coefficients of Variation. Commun. Stat. Simul. Comput., 35, 715-726.

[4] Conover, W.J. et al. (1981). A Comparative Study of Test for Homogeneity of Variances, with Applications to the Outer Continental Shelf Bidding Date. Technometrics, 23, 351-361. 
[5] Forkman, J., Verrill, S. (2008). The Distribution of McKay's Approximation for the Coefficient of Variation. Stat. Prob. Letters, 78, 10-14

[6] Hall, P., Wilson, S.R. (1991). Two Guidelines for Bootstrap Hypothesis Testing. Biometrics, $47,2,757-762$.

[7] Mahmoudvand, R., Hassani, H. (2009). Two New Confidence Intervals for the Coefficient of Variation in a Normal Distribution, Jour. of Appl. Statist., 36, 429-442.

[8] Nairy, K. S, Rao, K.A. (2003). Tests of Coefficients of Variation of Normal Population. Commun. in stat. Simul. comput., 32, 3, 641-661.

[9] Pang. W. et al. (2005). On Interval Estimation of the Coefficient of Variation for the Threeparameter Weibull, Lognormal and Gamma distribution: A simulation-based approach. Euro. Jour. Operat. Research, 164, 367-377.

[10] Robins, J. M., van der Vaart, A. W., Ventura, V. (2000). The Asymptotic Distribution of P-value in Composite Null Models. J. Amer. Statist. Assoc. 95, 1142-1156.

[11] Sachs, L. (1985). Applied Statistics. New York, Springer-Verlag. 